\title{
The genetic properties of homosexual copulation behaviour in Tribolium castaneum: artificial selection
}

\author{
L Castro $^{1}$, MA Toro ${ }^{1}$, C López-Fanjul ${ }^{2}$ \\ 1 Instituto Nacional de Investigaciones Agrarias, Departamento de Produción Animal, \\ Apartado 8111, 28080 Madrid; \\ 2 Facultad de Ciencias Biológicas, Departamento de Genética, \\ Universidad Complutense, 28040 Madrid, Spain
}

(Received 18 January 1993; accepted 2 December 1993)

\begin{abstract}
Summary - Artificial divergent selection for the rate of homosexual copulation (defined as the proportion of homosexual mountings performed by a male in a period of $30 \mathrm{~min}$ ) has been carried out for 2-3 generations in a population of Tribolium castaneum. A clear response was obtained in each of 4 replicates, corresponding to an overall realized heritability of $0.11 \pm 0.01$. No significant correlated response to selection was observed for the average number of mountings performed by a male during the testing period. Therefore, our results do not agree with evolutionary interpretations of insect homosexual copulation behaviour based on the existence of a negative genetic correlation between the degree of sexual discrimination and sexual activity. On the contrary, they strongly favour the hypothesis of sex recognition being absent in Tribolium casteneum.
\end{abstract}

homosexual copulation behaviour / artificial selection / Tribolium castaneum

Résumé - Les propriétés génétiques du comportement de copulation homosexuelle chez Tribolium castaneum : sélection artificielle. Une sélection divergente pour le taux de copulation homosexuelle (défini comme la proportion des montes homosexuelles réalisées par un mâle sur une période de $30 \mathrm{~min}$ ) a été réalisée sur $2-3$ générations dans une population de Tribolium castaneum. Une réponse nette a été obtenue dans chacune des 4 répétitions (1 lignée haute et 1 lignée basse par répétition), qui correspond à une héritabilité réalisée de $0,11 \pm 0,01$. Il n'a pas été observé de réponse indirecte à la sélection en ce qui concerne le nombre moyen de montes réalisées par un mâle sur la période d'épreuve. Ces résultats ne s'accordent pas avec les interprétations évolutionnistes du comportement de copulation homosexuelle chez les insectes, basées sur l'existence d'une corrélation génétique négative entre l'activité sexuelle et le degré de discrimination 
sexuelle. Au contraire, les résultats suggèrent très fortement l'absence de reconnaissance du sexe chez Tribolium castaneum.

comportement de copulation homosexuelle / sélection artificielle / Tribolium castaneum

\section{INTRODUCTION}

In Tribolium castaneum and $T$ confusum, high rates of homosexual copulation (defined as the proportion of homosexual mountings performed by a male in a period of $30 \mathrm{~min}$ ) have often been reported (Wool, 1967; Taylor and Sokoloff, 1971; Rich, 1972, 1989; Graur and Wool, 1982). They have routinely been considered as 'mistakes', or as a sort of pathological behaviour resulting from crowded laboratory conditions. Notwithstanding, Serrano et al (1991) found good agreement between observed rates of homosexual copulation in $T$ castaneum and those expected, assuming random pair contacts between potential mates, when both sexes equally oppose being mounted. This result has been shown to apply in a variety of experimental situations, indicating that $T$ castaneum males are indiscriminate with respect to sex.

The rate of homosexual copulation has been shown to be genetically variable in a laboratory population (Consejo population), by means of a diallel analysis of inbred lines and their $F_{1}$ crosses (Serrano et al, 1991). Nevertheless, no attempt was made to identify the forces maintaining that variation. Thornhill and Alcock (1983) have proposed an evolutionary interpretation of insect homosexual copulation behaviour, considered as a by-product of perception errors. Assuming that the main cost of reproduction is the time consumed by the male in discerning the sex of potential mates, increasing the number of mountings per unit time, at the risk of making some mistakes, may be more advantageous than carefully choosing a partner of the right sex. This implies the existence of a positive genetic correlation between the rate of homosexual copulation and mating activity, but estimates of this parameter are not available.

In the present work, artificial divergent selection was carried out for the rate of homosexual copulation to further study the genetic variation of the character. In addition, the genetic relationship between homosexual copulation and sexual activity has been explored.

\section{MATERIALS AND METHODS}

The Consejo population was captured near Madrid and has been maintained in a cage in this laboratory since 1964. All lines in this experiment were kept at $70 \%$ relative humidity and $33^{\circ} \mathrm{C}$. The culture medium consisted of $95 \%$ whole wheat flour and $5 \%$ dried, powdered brewer's yeast. Pupae were sexed by examination of the genital lobes. Male pupae were kept individually and female pupae stored at low densities, until tested (12-19 d after adult emergence) or used otherwise. 
Mating cannot be observed within the culture medium as beetles show a strong photonegative reaction. In this situation, our observations were made as follows. Two males (identified by a white or a yellow spot of paint on the thorax) were placed in a glass vial $(3 \mathrm{~cm}$ diameter $\times 3.5 \mathrm{~cm}$ height) with a filter paper floor, together with 2 virgin females from the unselected base population. For each male, the number of homosexual and heterosexual mountings were recorded during $30 \mathrm{~min}$ on each of 5 consecutive days, the females being substituted by a new pair each day. Ten such vials were observed simultaneously. Only those homosexual matings in which the mounting males showed symptoms of being sexually aroused (extension of aedagus) were recorded as such. Thus, the rate of homosexual copulation can be calculated for each male as the ratio between the number of homosexual mountings and the total number of mountings recorded (homo- and heterosexual).

Divergent selection for the rate of homosexual copulation was carried out in 4 non-contemporary replicates, although the high and low lines of the same replicate were always handled simultaneously. Individual selection was practised in each line on males. In all lines, the best 5 males were selected out of 40 tested, and individually mated to females taken at random from the same line and generation (full-sib matings were avoided). Each mating contributed 8 males and 8 females to the next generation.

\section{RESULTS}

The mean and the coefficient of variation of the rate of homosexual copulation, as well as its daily repeatability, are shown in table I for the Consejo population.

Table I. Parameters of the base population for the rate of homosexual copulation.

\begin{tabular}{lccc}
\hline Mean & Coefficient of variation (\%) & Daily repeatability (\%) & Heritability (\%) \\
\hline $29.5 \pm 0.74$ & 57.7 & $11.3 \pm 5.3$ & $11 \pm 1$ \\
\hline
\end{tabular}

* Overall realized heritability over the first $2-3$ generations of selection.

The rates of homosexual copulation of males identified with either a white or a yellow spot of paint did not significantly differ $\left(\chi_{1}^{2}=0.004\right)$. No significant heterogeneity was found between observations of the mating behaviour of males during 5 consecutive days $\left(\chi_{4}^{2}=8.4\right)$; a learning effect can therefore be discarded in our experimental conditions. This is not surprising as an intervening period of $23 \mathrm{~h}$ elapsed between consecutive tests. Obviously, this result cannot be extrapolated to laboratory or wild populations where mountings would occur continuously.

The average rate of homosexual copulation plotted against generation number is presented in figure 1 for the individual replicates. The difference between the performance of the upward and downward selected lines was statistically significant at any generation in all replicates. On average, a considerable divergence of $11.9 \%$ \pm 1.2 was obtained after only $2-3$ generations of selection. These results clearly demonstrate the existence of genetic variation for the selected trait. 

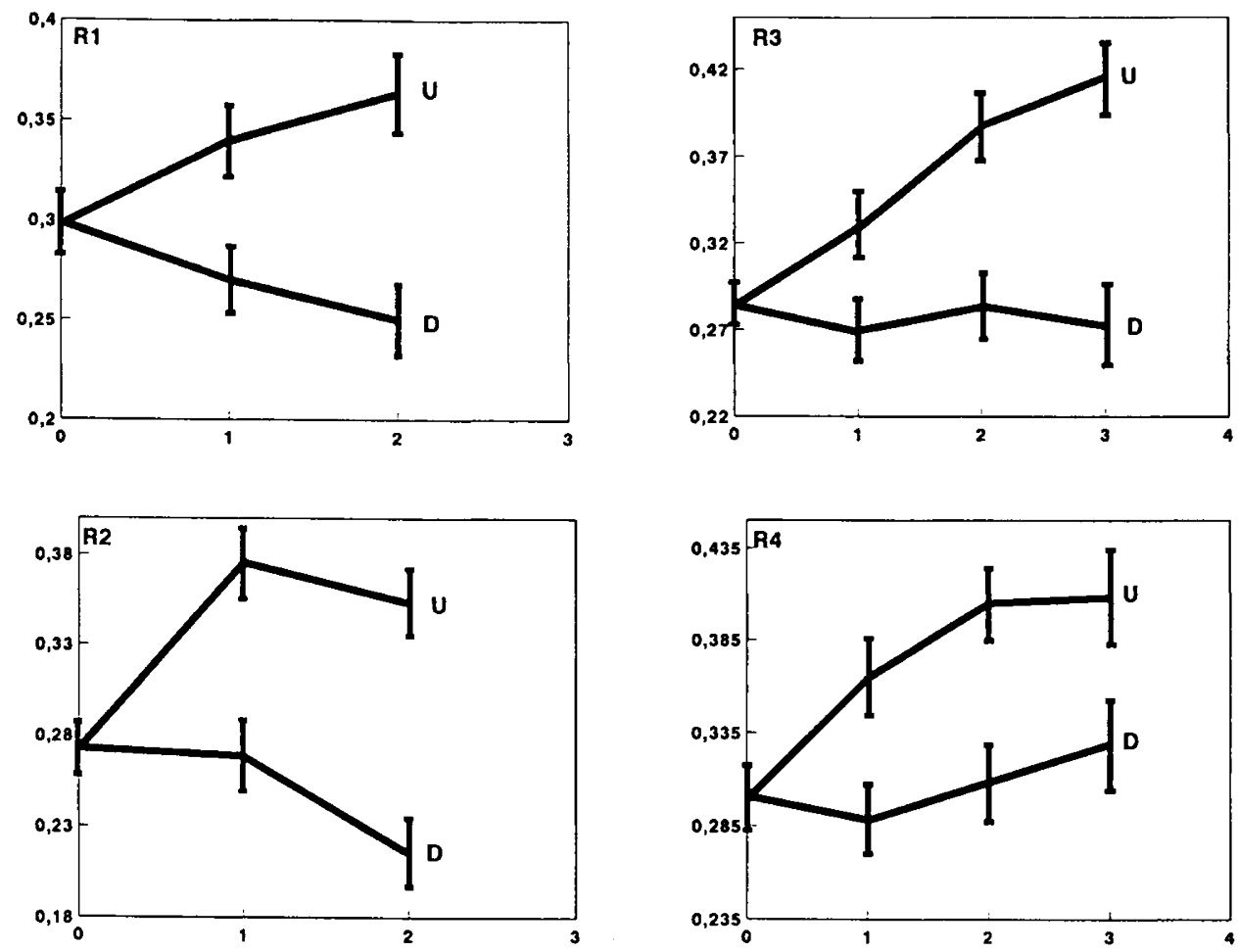

Fig 1. Mean rate of homosexual copulation ( \pm standard error) plotted against generation number, for each selected replicate ( $\mathrm{U}=$ upward, $\mathrm{D}=$ downward).

The response to selection was approximately symmetrical in replicates 1 and 2 (about $6 \%$ in each direction), and patently asymmetrical in replicates 3 and 4 , where much larger responses (11-13\%) were observed in the high line but no response was detected in the low line. On average, a much larger response was attained in the upward direction. After the data were arcsine transformed (not shown) asymmetry was still present, indicating that it cannot be accounted for by scale effects. Overall realized heritabilities (standard errors corrected for drift; Hill, 1972a,b) were $0.17 \pm 0.02$ (upwards), $0.08 \pm 0.01$ (downwards) and $0.11 \pm 0.01$ (divergence).

The average number of mountings (homo- and heterosexual) performed by a male in $30 \mathrm{~min}$ is shown in table II for the initial and final generation of selection for all lines. Male sexual activity increased slightly with selection. However, this occurred irrespective of the direction of selection and therefore this change can be better ascribed to an unidentified time trend. This is consistent with a higher value $(3.68 \pm 0.20)$ obtained by Serrano et al (1991) with the same base population. At the final generation, no significant differences were found between the male sexual activity of upward and downward selected lines in any replicate. This indicates that 
the genetic correlation between this trait and the rate of homosexual copulation must be small or non-existent.

Table II. Average number of mountings (homo- and heterosexual) performed by a male in $30 \mathrm{~min}^{*}$ in the initial and final generations of selection, for the upward (U) and downward (D) selected lines in each replicate.

\begin{tabular}{lccc}
\hline Replicate & Initial generation & \multicolumn{2}{c}{ Final generation } \\
& & $U$ & $D$ \\
\hline 1 & $3.08 \pm 0.16$ & $3.57 \pm 0.23$ & $3.43 \pm 0.21$ \\
2 & $3.44 \pm 0.15$ & $3.60 \pm 0.22$ & $3.31 \pm 0.19$ \\
3 & $2.89 \pm 0.13$ & $3.30 \pm 0.16$ & $3.51 \pm 0.19$ \\
4 & $2.91 \pm 0.13$ & $3.52 \pm 0.23$ & $3.53 \pm 0.24$ \\
Average & $3.08 \pm 0.07$ & $3.50 \pm 0.11$ & $3.45 \pm 0.10$ \\
\hline
\end{tabular}

The phenotypic correlations between the rates of homosexual copulation of the 2 males tested together in the same vial was negative but negligible $(-0.11$; confidence interval $-0.05,-0.17)$. In parallel, the phenotypic correlation between the average number of mountings in $30 \mathrm{~min}$ and the rate of homosexuality of a male was positive but small $(0.19$; confidence interval $0.13,0.25)$. Both correlations have been calculated from pooled data (base population and selected lines). Nonsignficant estimates of these correlations were obtained by Serrano et al (1991).

\section{DISCUSSION}

Our experimental design consisted of 4 non-contemporary replicates but, in each of them, artificial selection was carried out in both directions on a strictly contemporary schedule. Thus, the data allow us to evaluate correctly total response (divergence) to selection, either direct or correlated. Strictly speaking, however, no conclusions could be drawn on the responses attained in each direction, since possible environmental trends and/or asymmetry of response could not be properly accounted for in the absence of a control line. General between-replicate agreement has been found for the questions of interest. In all replicates, a significant divergence for the rate of homosexual copulation was obtained by practising direct selection for this trait. This result clearly shows that the differences between individuals for the rate of homosexual copulation are partly genetic in the Consejo population, reinforcing previous evidence from diallel analysis of data from highly inbred lines derived from the same populations (Serrano et al, 1991).

The evolutionary hypothesis proposed by Thornhill and Alcock (1983) assumes that individuals showing a lower degree of sexual discrimination will also have a higher sexual activity and may thus achieve higher reproductive fitness. In other words, the degree of sexual discrimination (represented by the rate of homosexual copulation) and sexual activity (represented by the average number of mountings performed by a male per unit of time) must be genetically correlated. Our results do not support this hypothesis. First, a small non-significant correlated response 
for the number of mountings was observed when selecting up and down for the rate of homosexual copulation, both in each replicate and overall. This implies that the genetic correlation between these traits will also be small and not significant in the Consejo base population, although a numerical estimate cannot be calculated from our data. On the other hand, the corresponding phenotypic correlation was small but significant. However, this parameter contains little information on the genetic correlation and, in our case, its value is compatible with a zero genetic correlation provided the heritability of the number of mountings is less than 0.95 . Second, males from the upward selected lines (lower degree of sexual discrimination) mounted fewer females than those from downward selected lines (higher degree of discrimination) and, therefore, the latter are fitter in this respect.

The results obtained by Serrano et al (1991) indicate that sex recognition is absent in $T$ castaneum and, therefore, homosexuality cannot be simply explained in terms of perception errors. In the model proposed by these authors, the probability of homosexual mountings $\left(p_{2}\right)$ is a function only of the composite parameter $\alpha$, which represents the balance between the male's persistence in mounting and the differential resistance offered by males and females to being mounted. In this situation, the behaviour of mounting males as well as the differential reactions of the individuals being mounted (traditionally regarded as passive partners) are taken into account. More formally, $p_{2}=1 /(1+4 \alpha)$, where $\alpha=y / x$; and $x$ and $y$ are the probabilities of homosexual and heterosexual pair contacts between individuals resulting in mounting respectively. Thus, the response to divergent selection can be analysed in terms of the corresponding changes in the ratio $\alpha$ of these 2 probabilities. A value of $p_{2}=31.6 \% \pm 1.9$ was estimated by Serrano et al (1991) in the Consejo base population. Our estimate $(29.5 \% \pm 0.74)$ does not significantly differ from the former though it is slightly lower and significantly smaller than 0.33 , the expected value corresponding to random pair contacts between sexually indiscriminate individuals, when both sexes being mounted equally reject.

A response in the upward direction can be achieved by lowering the male's general sexual vigour, in particular by decreasing the male's resistance to being mounted relative to that of the unselected (non-inbred) females used for testing (although not necessarily with respect to females from the same selected line). This does not need to affect the total number of mountings performed by a male per unit of time, as the increasing difficulties in mounting females may be compensated by a larger number of homosexual mountings. In fact, the average male's sexual activity was not affected by selection in either direction. In the same testing conditions of this experiment, inbreeding has been shown to enhance the rate of homosexual copulation by depressing a male's vigour (Serrano et al, 1991). Therefore, upward selection could also act through the same mechanism. In this situation, however, response to downward selection will be more difficult to attain, as it will require an increase of the sexual vigour of males above that achieved by natural selection in the base population. Thus, the asymmetrical response found in this experiment is consistent with this hypothesis. 


\section{ACKNOWLEDGMENTS}

The authors thank A Gallego, A García-Dorado and B Jódar for helpful comments on the manuscript, and RG Ruano for laboratory assistance.

\section{REFERENCES}

Graur D, Wool D (1982) Dynamics and genetics of mating behavior in Tribolium castaneum (Coleoptera: Tenebrionidae). Behav Genet 12, 161-179

Hill WG (1972a) Estimation of realised heritabilities from selection experiments. I. Divergent selection. Biometrics $28,747-765$

Hill WG (1972b) Estimation of realised heritabilities from selection experiments. II. Selection in one direction. Biometrics $28,767-780$

Rich E (1972) Homosexual behavior related to a melanic mutant in Tribolium castaneum. Tribolium Inf Bull 15, 97

Rich E (1989) Homosexual behavior in three melanic mutants of Tribolium castaneum. Tribolium Inf Bull 29, 99-101

Serrano JM, Castro L, Toro MA, López-Fanjul C (1991) The genetic properties of homosexual behavior in Tribolium castaneum: diallel analysis. Behav Genet 21, 547558

Taylor C, Sokoloff A (1971) A review of mating behavior in Tribolium. Tribolium Inf Bull $14,88-91$

Thornhill R, Alcock J (1983) The Evolution of Insect Mating Systems. Harvard University Press, Cambridge, MA, USA

Wool D (1967) Some observations on mating frequencies in Tribolium castaneum strains. Tribolium Inf Bull 10, 182-186 